\title{
Multigene Methylation Analysis And The Noninvasive Diagnosis Of Prostate Cancer From Body Fluids
}

\section{Dumache Raluca, MD, PhD, MSc}

Department of Forensic Medicine,

Victor Babes University of Medicine and Pharmacy, Timisoara, Romania

\section{Tudor Anca, PhD}

Department of Medical Informatics,

Victor Babes University of Medicine and Pharmacy, Timisoara, Romania

\section{Ionescu Daniela, MD, PhD}

Department of Toxicology,

Victor Babes University of Medicine and Pharmacy, Timisoara, Romania

\section{Rogobete Alexandru Florin, MSc}

Victor Babes University of Medicine and Pharmacy, Timisoara, Romania

\section{Bardan Razvan, MD, PhD}

Department of Urology,

Victor Babes University of Medicine and Pharmacy, Timisoara, Romania

\section{doi: 10.19044/esj.2016.v12n9p412ＵRL:http://dx.doi.org/10.19044/esj.2016.v12n9p412}

\section{Abstract}

Introduction During prostatic carcinogenesis, DNA hypermethylation occurs, thus representing a promising biomarker for the early detection of this malignancy. In our study, we aim to determine the usefulness of a molecular and multigene test for prostate cancer. However, this is based on the quantitative methylation-specific polymerase chain reaction (qMSP) of three genes from voided urine specimens by noninvasive methods.

Materials and Methods In this study, the voided urine specimens were collected from 89 patients with prostate cancer and 69 controls. Genomic DNA was isolated and subjected to bisulfite modification. Consequently, we tested the methylation status of genomic DNA of three genes, namely: GSTP1, APC, and MDR1. This was done using the quantitative methylationspecific PCR method. Therefore, the obtained results were correlated with the clinicopathologic findings.

Results Promoter methylation of GSTP1 gene in voided urine samples was found in 87 out of $89(97.8 \%)$ PCa patients and in 13 out of $62(21 \%) \mathrm{BPH}$ 
men. In APC gene, methylated levels have been found in 61 out of 89 $(68.5 \%)$ PCa patients and in 8 out of $62(12.9 \%)$ BPH men. MDR1 gene was found to be hypermethylated in 60 out of $89(67.4 \%) \mathrm{PCa}$ patients and in 4 out of $62(6.5 \%) \mathrm{BPH}$ men. In addition, we obtained a sensitivity of $88.99 \%$ and a specificity of $85.5 \%$ for the multigene panel. The AUC in this case was 0.927.

Conclusion The analysis of a multigene panel of three methylated genes in prostate cancer by qMSP, can be used to distinguish between men with malignant and benign prostatic diseases from voided urine specimens. Also, it can be used for the follow-up of those men who are presenting increased risk of prostate cancer by noninvasive methods.

Keywords: Prostate cancer (PCa), benign prostatic hyperplasia (BPH), quantitative methylation-specific polymerase chain reaction (qMS-PCR), glutathione S-transferase P1 (GSTP1), multidrug resistance 1 (MDR1) gene, adenomatous polyposis coli (APC) gene

\section{Introduction}

Worldwide, prostate cancer $(\mathrm{PCa})$ represents the second leading cause of mortality in men. Each year, it is responsible for more than 29.000 deaths (Siegel et al., 2013). However, this was because in its early stage, it evolves as asymptomatic. Generally, it is discovered in a late stage. Therefore, there is an urgent need for its introduction in the clinic of an algorithm. These include several sensitive biomarkers which can detect the disease at its early stage, by noninvasive techniques from body fluids.

Currently, the diagnostic of prostate cancer is done based on a combination between serum (PSA), digital rectal examination (DRE), and histological examination of transrectal ultrasound (TRUS)-guided biopsy. Since the introduction of serum prostate specific antigen (PSA) as a routine biomarker, in addition to the DRE, it has led to the increase of organ confined prostate cancer cases (Brawer, 2000). The use of serum PSA as a predictive biomarker in prostate cancer has some limitations. These limitations include low specificity of serum PSA which leads to frequent unnecessary sextant biopsies. In patients with the serum PSA levels in the range of $3 \mathrm{ng} / \mathrm{ml}$ and $10 \mathrm{ng} / \mathrm{ml}$, known as the grey zone, the serum PSA test has low specificity to detect prostate cancer. Thus, this results in an increased rate of negative biopsies between $60 \%$ to $75 \%$; hence, this will undergo unnecessary sextant biopsies (Schroder et al., 2014). The increased levels of serum PSA above $3.00 \mathrm{ng} / \mathrm{ml}$ are considered to be associated with prostate cancer, while the increased levels of serum PSA are also present in benign prostatic diseases such as benign prostatic hyperplasia (BPH), which is observed in elderly (Thompson et al., 2004). Also, another inconvenience is 
due to the TRUS-biopsies which can miss some tumoral foci or the presence of higher-grade tumor (Haffner et al., 2015). In the last twenty years, due to the developments in the field of molecular genetics, many genetic and epigenetic alterations have been discovered to be involved at the molecular level in the prostatic carcinogenesis. DNA methylation represents an important epigenetic modification which occurs during the process of carcinogenesis. Also, it involves the transfer of a methyl group to the 5' position of the cytosine ring of the cytosine phosphate guanosine $(\mathrm{CpG})$ dinucleotides through the DNA methyltransferases. DNA hypermethylation has been associated with carcinogenesis. In addition, some studies have shown that it occurs at the early stages of the carcinogenesis, making it an ideal biomarker for early cancer detection (Chiam et al., 2012). Although previous studies have shown that some methylated loci such as GSTP1, RASSF1A, and PTGS2 can be used as biomarkers for diagnostic and/or prognostic in prostate cancer (Roupret et al., 2007), recent studies have suggested that using methylated gene panels for the diagnosis and/or prognosis of prostate cancer patients can improve the sensibility and specificity of the test compared to serum PSA. However, this fact is important in deciding the need for a second prostate biopsy in patients with a first negative biopsy (Partin et al., 2014; Stewart et al., 2013).

In the present study, we would demonstrate that the use of a panel of 3 methylated biomarkers (glutathione S-transferase P1 (GSTP1), adenomatous polyposis coli (APC), and multidrug resistance 1 (MDR1) can serve as an adjunct to serum PSA in the early diagnostic of prostate cancer. Subsequently, it can improve in distinguishing between malignant and benign lesions by noninvasive methods.

\section{Materials and Methods}

\section{Patients, Sample Collection, and DNA Extraction}

In our study, we included a number of 89 men with clinically localized prostate adenocarcinoma. They were primarily treated with radical prostatectomy at the Department of Urology from "Pius Brinzeu" Clinical Emergency Hospital in Timisoara, Romania. Thus, this took place between 2014-2015. The cases were identified due to the increased levels of serum prostate specific antigen during routine analysis, and were confirmed by sextant prostate biopsy. All the biopsies were performed transrectally under ultrasound guidance. Also, a number of 62 men were included as controls in our study. They were confirmed by serum PSA levels in the range of 3.0 $\mathrm{ng} / \mathrm{ml}$ to $9.9 \mathrm{ng} / \mathrm{ml}$, and it has an initial negative prostate biopsy result. In the control groups, men with no history of genitourinary malignancy were included. 

follows:

The eligibility criteria for prostate cancer patients' selection were as

1. Only patients with clinical tumor stage I or II were included in the study.

2. Only patients who had no clinical evidence of lymph node involvement or distant metastasis were included.

3. Only patients who had no previous treatment with hormonal therapy or radiation therapy before urine sample collection were included.

For the controls, the eligibility criteria were as follows:

1. Serum PSA levels between $3.0 \mathrm{ng} / \mathrm{ml}$ and $9.9 \mathrm{ng} / \mathrm{ml}$.

2. A negative prostate biopsy result.

\section{Urine Sample Collection}

The urine samples $(20-30 \mathrm{~mL})$ were collected following a digital rectal examination (DRE) which consisted of 3 strokes per prostatic lobe. Therefore, this was performed by the urologist. After the urine specimens were obtained, they were stored at 2-8 C and were processed within 4 hours. The urine samples were processed according to the procedures for the whole urine as described by Groskopf et al. (2006). On the other hand, the urine samples were processed for urinary sediments as described by Hessels et al. (2003).

In measuring the levels of serum PSA in the subjects included in our study, we used the enzyme linked-immunosorbent assay (ELISA) method. Thus, the measurements were done using an Advia Centaur immunoassay system (Bayer Healthcare, USA).

\section{Genomic DNA Extraction from Urine Samples}

Consequently, we extracted the genomic DNA from urine samples using the Urine DNA Isolation Kit (Norgen, Biotek Corporation, Canada). After extraction, we quantified the obtained genomic DNA by measuring it with a Qubit 3.0 Fluorometer (Invitrogen, USA).

\section{Bisulfite Modification}

Extracted DNA samples were subjected to sodium bisulfite conversion using the EpiTect Fast Bisulfite Conversion Kit (Qiagen, Germany) by following the manufacturer's instructions.

\section{Methylation Analysis}

We used the bisulfite-treated DNA as a template for the quantitative fluorescence real-time methylation-specific PCR (qMSP) as it was previously described by Hoque (Hoque et al., 2007). We investigated the abberant promoter methylation using qMSP for three gene promoter regions: 
GSTP1, MDR1, and APC. For the methyl quantification, the polymerase chain reaction was done using the bisulfite specific primers to the $\beta$-actin gene. The primers and probes have been designed using the MethyPrime Soft to amplify the bisulfite converted promoter of the genes. Fluorescence based real-time PCR assay was carried out in a reaction volume of $25 \mu \mathrm{L}$ consisting of $0.25 \mu \mathrm{L}$ of each primer, $12.5 \mu \mathrm{L}$ qPCR Master Mix (Fermentas, Lithuania), $2 \mu \mathrm{L}$ bisulfite converted DNA, $0.5 \mu \mathrm{L}$ of each sample, and $5 \mu \mathrm{L}$ of distilled water. However, each sample was run in duplicate. As a positive control, we used universally methylated DNA (Chemicon, USA). All amplifications were carried out in a 96-well plates (Applied Biosystems, USA) on a 7500 Real -Time PCR System (Applied Biosystems, USA). The conditions for the amplification were as follows: $95^{\circ} \mathrm{C}$ for 10 minutes followed by 45 cycles of $95^{\circ} \mathrm{C}$ for 15 minutes, and $60^{\circ} \mathrm{C}$ for 1 minute.

\section{Ethical Aspects regarding the Study}

The Institutional Committee of Ethics from Victor Babes University of Medicine and Pharmacy in Timisoara, Romania, approved our study. Therefore, it was carried out in accordance with the World Medical Association Declaration of Helsinki for Human Research statements revised in 2013. First, we informed the subjects included in the study regarding the protocol of the study. After their acceptance to be part of the study, they gave their written informed consent.

\section{Statistical Analysis}

The correlations between the methylation levels and the clinicopathological variables were evaluated using the nonparametric MannWhitney tests for continous variables.

To discriminate malignant versus benign cells, we used the variables such as sensitivity and specificity of methylation. Consequently, this variable were determined by the receiver operator curve (ROC) analysis. The discriminatory power of the test was given by the area under the curve (AUC).

In determining the sensitivity and specificity of the DNA methylation in differentiating between the two groups, we calculated the receiver operating curve (ROC). Furthermore, we used Pearson's correlation to evaluate the relation between methylation level of GSTP1, APC, and MDR1 gene with the clinico-pathological parameters.

Statistical analysis were performed using SPSS ver. 20.0 (SPSS Inc.,Chicago,IL,USA). Also, we considered a p-value $<0.05$ to be statistically significant. 


\section{Results}

The baseline characteristics of the patients included in our study is presented in Table 1. In addition, the distribution between the methylated and unmethylated genes is presented in Figure 1.

Table 1. Characteristics of the prostate cancer cases and control cases included in the study

\begin{tabular}{|c|c|c|c|c|c|c|c|c|}
\hline Variables & Sample & $\mathbf{N}$ & Mean & $\begin{array}{l}\text { Std. } \\
\text { Deviation }\end{array}$ & $\begin{array}{l}\text { Std. } \\
\text { Mean }\end{array}$ & Error & Minimum & Maximum \\
\hline \multirow[t]{2}{*}{ AGE } & $\begin{array}{l}\text { PROSTATE } \\
\text { CANCER }\end{array}$ & 89 & 63.82 & 11.833 & 1.254 & & 40 & 85 \\
\hline & CONTROL & 62 & 58.74 & 15.034 & 1.909 & & 25 & 88 \\
\hline \multirow[t]{2}{*}{ PSA } & $\begin{array}{l}\text { PROSTATE } \\
\text { CANCER }\end{array}$ & 89 & 17.27 & 5.497 & 0.583 & & 10 & 35 \\
\hline & CONTROL & 62 & 6.00 & 2.188 & 0.278 & & 2 & 10 \\
\hline
\end{tabular}

Figure 1. Distribution between methylated and unmethylated genes

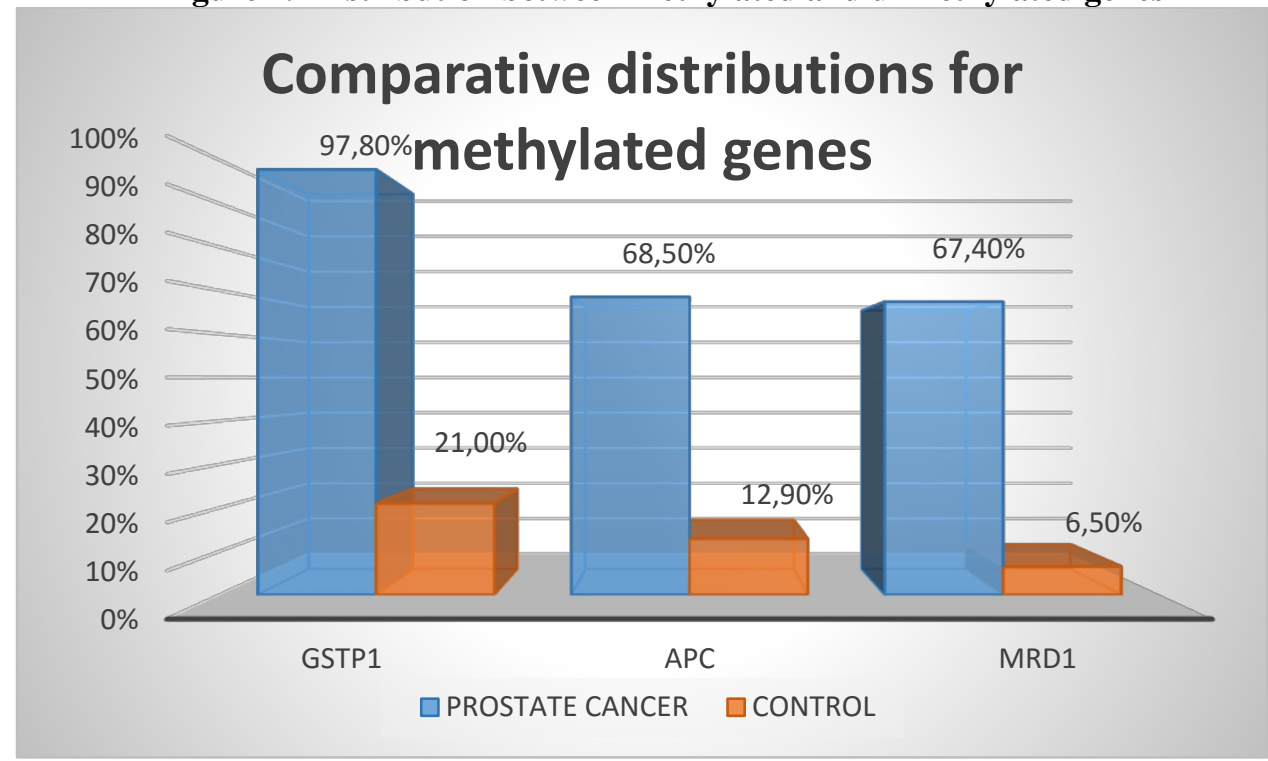

However, we made correlations between different parameters and the methylation levels of the three genes as follows:

a. Correlations between methylation levels, preoperative serum prostate-specific antigen (PSA) levels, and age

Preoperative serum PSA levels were increased in prostate cancer patients compared to those with benign prostatic hyperlasia (MannWhitney test; $p=0.028, \alpha=0.05$ ).

Also, according to the Spearman rank-correlation test, there was a significant correlation between the serum PSA levels and the methylation levels of the three genes in the prostate cancer group (Spearman coefficient $=0.872 ; \mathrm{p}<0.001)$. 
b. Correlations between methylation levels of GSTP1, APC, and MDR1 in prostate cancer patients versus controls

The methylation levels of gluthatione S-transferase P1 gene was significantly increased in the prostate cancer group when compared to the benign prostatic hyperplasia group $\left(\chi^{2}\right.$ test $\left.; \mathrm{p}<0.001\right)$. Glutathione S-transferase P1 gene was found hypermethylated in 87 out of $89(97.8 \%)$ patients with prostate cancer, and in 13 out of 69 $(21 \%)$ of those with benign prostatic hyperplasia. Using the receiver operating curve (ROC), we found that GSTP1 gene can discriminate between the prostate cancer patients and BPH patients. This is with a sensitivity of $87.75 \%$ and a specificity of $79.03 \%$, respectively. The area under the curve (AUC) of the GSTP1 gene was 0.915. Thus, the AUC is presented in Figure 2 below.

Figure 2. Receiver operating curve (ROC) analysis of GSTP1 gene hypermethylation levels in prostate cancer cases ROC Curve

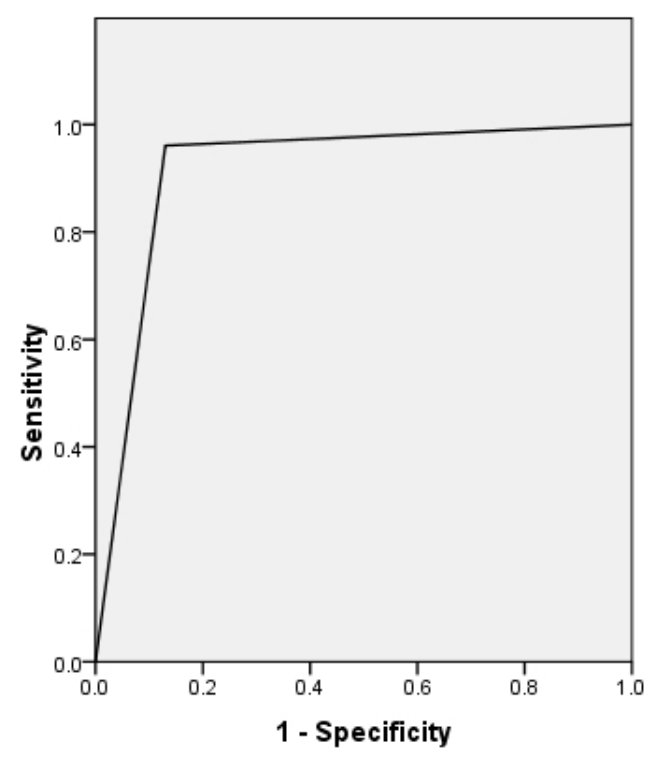

Adenomatous polyposis coli (APC) was found methylated in 61 out of $89(68.5 \%)$ prostate cancer patients and in 8 out of $69(12.9 \%)$ controls. The ROC in the case of APC gene presented a sensitivity of $68.54 \%$ and a specificity of $87.1 \%$ in discriminating $\mathrm{PCa}$ from controls. The AUC was 0.771. Thus, the AUC is presented in Figure 3 below. 
Figure 3. Receiver operating curve (ROC) analysis of APC gene hypermethylation levels in prostate cancer cases ROC Curve

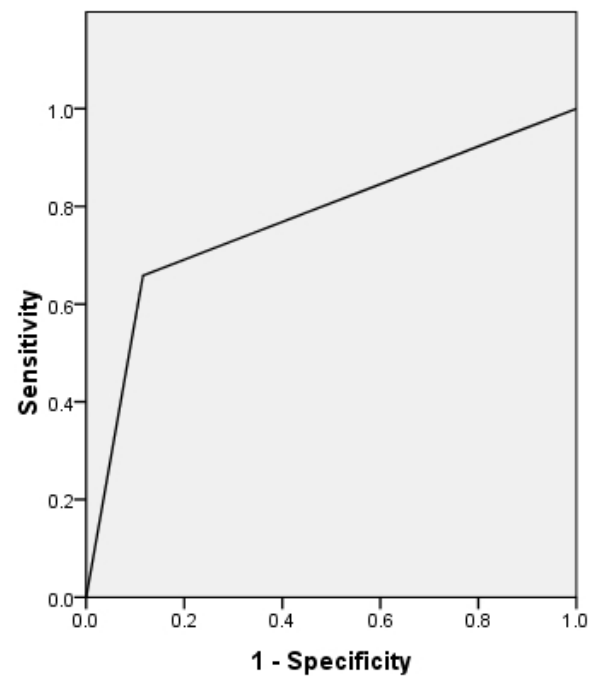

Multidrug resistance 1 (MDR1) was found hypermethylated in 60 out of $89(67.4 \%)$ prostate cancer patients and in 4 out of $62(6.5 \%)$ controls. The receiver operating curve had a sensitivity of $67.42 \%$ and a specificity of $93.55 \%$, respectively. The area under the curve (AUC) was 0.802. Hence, the AUC is presented in Figure 4 below.

Figure 4. Receiver operating curve (ROC) analysis of MRD1 gene hypermethylation levels in prostate cancer cases

ROC Curve

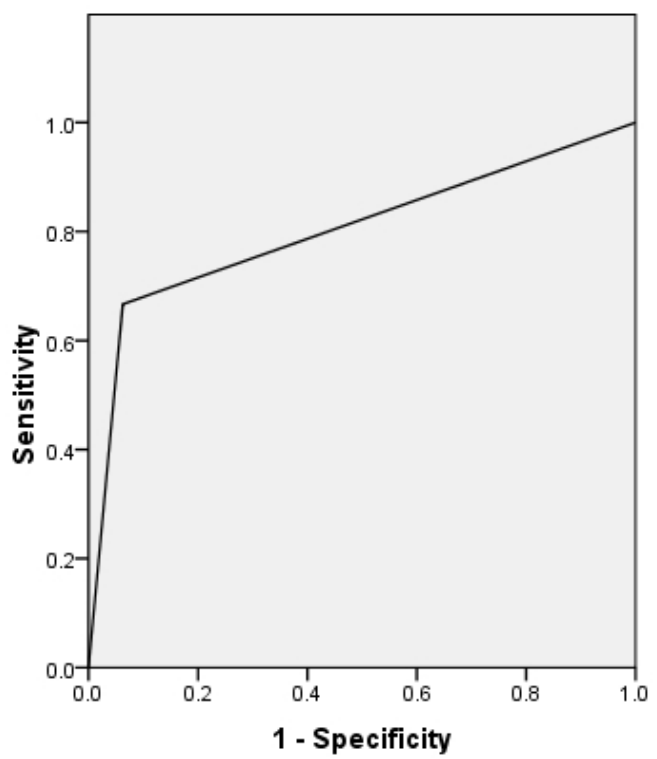


Figure 5. Multigene analysis of GSTP1, APC, and MDR1 genes

ROC Curve

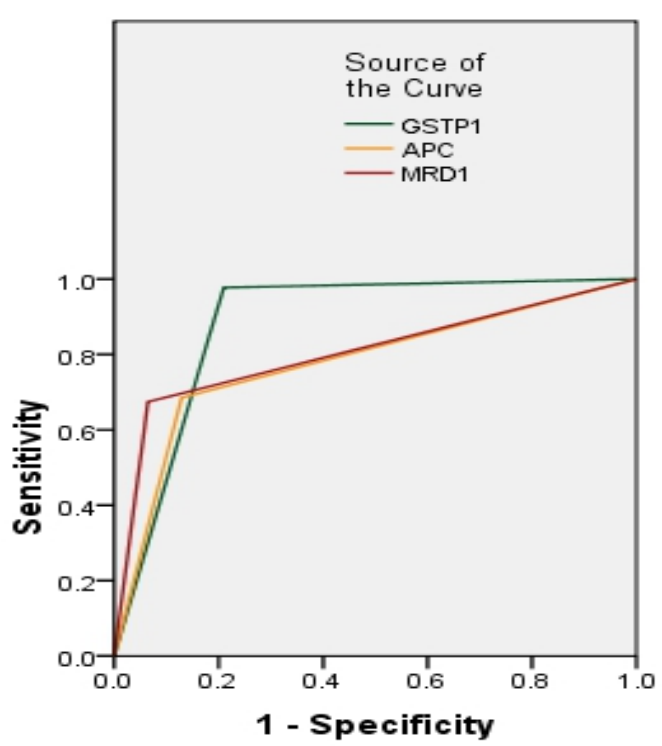

The multigene panel has a sensitivity of $\mathbf{8 8 . 8 9} \%$ and a specificity of $\mathbf{8 5 . 5 \%}$, respectively. The area under the curve (AUC) in this case was 0.927 .

\section{Conclusion}

Currently, the combination between serum PSA and DRE represents the most used methods for the clinicians for identifying those patients who present the risk of developing prostate cancer. The serum PSA test presents a sensitivity and specificity between $70 \%$ to $90 \%$ and $20 \%$ to $40 \%$, respectively (Brawer, 1999). Brawer et al, in their study, obtained an area under the curve (AUC) in a range between 0.55 to 0.70 . This was based on the ability of serum PSA to identify those men who present prostate cancer. Due to these disadvantages, there is an urgent need for validating new biomarkers which can aid in early detection.

However, regarding the development and progression of prostate carcinogenesis, many studies have been done related to the involvement of the DNA methylation (Goering et al., 2012). Glutathione S-transferase P1 (GSTP1) gene promoter hypermethylation has been reported to be the most frequent epigenetic modification which occurs during prostate carcinogenesis. At present, it occurs in about $70 \%$ of high-grade prostatic intraepithelial neoplasia (HGPIN) lesions and in about $90 \%$ of neoplastic tissues samples when compared to normal benign tissue (Nakayama et al., 2003). 
The study of Roupret et al. (2007) shows that using an epigenetic multigene panel made of 4 genes (GSTP1, RASSF1A, RAR $\beta 2$, and APC), we demonstrated that this model could differentiate between malignant and nonmalignant cases with a sensitivity of $86 \%$ and a specificity of $89 \%$, respectively. Consequently, Hoque et al examined the promoter methylation in 9 genes from urine sediment to distinguish between prostate cancer patients and control cases. Their results are correlated with the methylation levels from the corresponding primary tumors. From the panel of 9 genes, a combination of only 4 genes (p16, ARF, MGMT, and GSTP1) could detect prostate cancer with a sensitivity of $87 \%$ and a specificity of $100 \%$ (Hoque et al., 2005).

In our study, we determined the methylation levels of three methylated genes from urine samples: GSTP1, APC, and MRD1. Using the receiver operating curve (ROC), we found that each gene can discriminate between the prostate cancer patients and BPH patients based on the obtained sensitivity and specificity for each. Moreover, in this study, we performed a methylation analysis of the combination of the three genes. In addition, we obtained a sensitivity of $88.9 \%$ and a specificity of $85.5 \%$, respectively.

The obtained AUC for the multigene panel was 0.927. The limitations of our study can be represented by the small number of patients included in the study and the short period of time for the study. Therefore, further studies of larger study groups are needed to confirm our preliminary data.

In the future, this panel of methylated genes can be used for the early diagnosis of prostate cancer by noninvasive methods. Therefore, its aim is to reduce the need for repeated prostate biopsy, and to follow-up men that shows higher risk of prostate cancer.

\section{References:}

Brawer (2000). Screening for prostate cancer. Seminars in Surgical Oncology 18;29-36.

Brawer (1999). Prostate-specific antigen: current status. CA Cancer Journal for Clinicians 49(5): 264-281.

Chiam et al. (2012). Epigenetic biomarkers in prostate cancer: current and future uses. Cancer Lett 342: 248-56

Goering et al. (2012). DNA methylation changes in prostate cancer. Methods in Molecular Biology 863: 47-66.

Groskopf et al. (2006). APTIMA PCA3 molecular urine test: development of a method to aid in the diagnosis of prostate cancer. Clin Chem 52:1089-95 Haffner et al. (2015). Diagnostic challenges of clonal heterogeneity in prostate cancer. J Clin Oncol Mar 1; 33(7): e38-40 
Hessels et al. (2003). DD3(PCA3)-based molecular urine analysis for the diagnosis of prostate cancer. Eur Urol 44: 8-15

Hoque et al. (2005). Quantitative methylation-specific polymerase chain reaction gene patterns in urine sediment distinguish prostate cancer patients from control subjects. J Clin Oncol 23: 6569-75.

Nakayama et al. (2003). Hypermethylation of the human glutathione Stransferase- $\pi$ gene (GSTP1) CpG island is present in a subset of proliferative inflammatory atrophy lesions but not in normal or hyperplastic epithelium of the prostate: a detailed study using laser-capture microdissection. American Journal of Pathology 163(3): 923-933

Partin et al. (2014). Clinical validation of an epigenetic assay to predict negative histopathological results in repeat prostate biopsies. J Urol 192: 1082-7

Roupret et al. (2007). Molecular detection of localized prostate cancer using quantitative methylation-specific PCR on urinary cells obtained following prostate massage. Clin Cancer Res 13:1720-5

Schroder et al. (2014). Screening and prostate cancer mortality: results of the European Randomised Study of Screening for Prostate Cancer (ERSPC) at 13 years follow-up. Lancet 384: 2027-35

Siegel et al. (2013). Cancer statistics 2013.CA Cancer J Clin 63:11-30

Stewart et al. (2013). Clinical utility of an epigenetic assay to detect occult prostate cancer in histopathologically negative biopsies: results of the MATLOC study. J Urol 189:1110-6.

Thompson et al. (2004). Prevalence of prostate cancer among men with a prostate-specific antigen level < or $=4.0 \mathrm{ng}$ per milliliter. N Engl J Med 350: $2239-46$ 